\title{
Gelatinase and oncofetal fibronectin secretion is dependent on integrin expression on human cytotrophoblasts*
}

\author{
P.Bischof', L.Haenggeli and A.Campana \\ Clinic of Infertility and Gynecologic Endocrinology, Department of Obstetrics and Gynecology, University of Geneva, \\ Geneva, Switzerland \\ ${ }^{1}$ To whom correspondence should be addressed at: Laboratoire d'Hormonologie Maternité, 1211 Genève 14 , Switzerland
}

Collagenolytic activity of cytotrophoblasts is stimulated by glycoproteins of the extracellular matrix and since this stimulation can possibly occur through integrins, we measured the gelatinolytic activity of villous and extravillous cytotrophoblasts according to the type of integrins expressed on these cells. Cytotrophoblasts were isolated from legal abortions, immunopurified with anti-CD45, separated according to their expression of histocompatibility-linked antigen (HLA)-G, $\alpha_{6}$ or $\alpha_{5}$ integrin subunits and cultured for 5 days on plastic or agarose. Fetal fibronectin, human chorionic gonadotrophin (HCG) and the gelatinolytic activity were measured in the culture supernatants. Following immunopurification with anti-CD45, the gelatinolytic activity of cytotrophoblasts was significantly higher than before, indicating that contaminating lymphomyeloid cells secreted gelatinolytic inhibitors. HLA-G positive cells secreted significantly more gelatinases than HLA-G negative cells but their HCG secretion was similar. Compared to $\alpha_{5}$ positive cells, $\alpha_{6}$ positive cytotrophoblasts secreted significantly more gelatinases, significantly less fibronectin but similar amounts of HCG. We conclude that during trophoblast invasion, extravillous cytotrophoblasts (HLA-G positive) expressing the $\alpha_{6}$ integrin subunit represent the invasive population of cells (high gelatinase and low fibronectin secretion). When expression of the $\alpha_{5}$ integrin subunit is turned on, their invasive behaviour ceases and they secrete low amounts of gelatinases and high concentrations of fibronectin.

Key words: fibronectin/gelatinase/integrins/trophoblast invasion

\section{Introduction}

Implantation and placentation in humans are dependent upon a tightly controlled invasion of the maternal endometrium by fetal trophoblast. Deregulation of this process in either direction leads to severe pathological conditions: insufficient invasion is associated with pre-eclampsia and inadequate fetal growth (Khong et al., 1986; Zhou et al., 1993), whereas unrestricted invasion leads to pre-malignant conditions such as hydatidiform mole.

After initial attachment of the blastocyst to the uterine lining, mononuclear cytotrophoblasts, which surround the embryonic disc, fuse to form syncytia (Kao et al., 1988). These multinucleated giant cells invade the pseudodecidualized endometrium (Weitlauf, 1988). Once the definitive placental villi are formed, some cytotrophoblasts of anchoring villi (which contact the uterine wall) acquire a transiently invasive phenotype and invade the decidualized endometrium, while the cytotrophoblasts of floating villi (in the extravillous space) remain attached to the villous basement membrane. Thus, cytotrophoblasts follow one of two existing differentiation pathways: villous cytotrophoblasts form a monolayer of polarized epithe-

* Part of the results described here were presented at the ESHRE meeting in Brussels in June 1994. lial stem cells which eventually differentiate by fusion to form a syncytial layer (Kao et al., 1988) which covers the entire surface of the villous. Cytotrophoblasts, in anchoring villi, either follow the differentiation pathway to form syncytia or break through the syncytium at selected sites to form multilayered columns of non-polarized cytotrophoblasts. These motile and highly invasive cells, also referred to as intermediate trophoblasts (Enders, 1968), are found as cytokeratin positive cells in the decidua, the intima of uterine blood vessels and the proximal third of the myometrium. The molecular mechanism which directs cytotrophoblasts into one or the other differentiation pathway is the subject of intensive research in many centres.

Villous and extravillous cytotrophoblasts are morphologically and functionally distinct (Enders, 1968; Damsky et al., 1992; Genbacev et al., 1993). They are both cytokeratin positive, indicating their common epithelial nature, but only intermediate cytotrophoblasts express a non-classical human leukocyte class I antigen (HLA-G) (Chumbley et al., 1993; Shorter et al., 1993) whereas only villous cytotrophoblasts have epidermal growth factor receptors (Mühlhauser et al., 1993). Furthermore, villous and intermediate cytotrophoblasts are in a different microenvironment. Villous cytotrophoblasts 
are bound to a basement membrane composed of collagen type IV, fibronectin and laminin whereas intermediate cytotrophoblasts, depending on their location (proximal or distal), are surrounded by a matrix composed either of modified laminin or fibronectin and collagen type IV respectively (Damsky et al., 1992).

Extracellular matrix components are known to influence adhesion, spreading, migration and differentiation of cells. The cells recognize their microenvironment through specific membrane receptors. Among the different adhesion molecules, integrins mediate essentially but not exclusively the interactions between cells and the glycoproteins of the extracellular matrix (Ruoslahti, 1991; Heino, 1993). Integrins are heterodimeric transmembrane glycoproteins composed of an $\alpha$ and a $\beta$ subunit. So far eight $\beta$ and $14 \alpha$ subunits have been identified, forming a family of about 20 receptors. Depending on the type of $\alpha / \beta$ combination the integrins will bind to one or another matrix glycoprotein, i.e. $\alpha_{5} \beta_{1}$ to fibronectin, $\alpha_{6} \beta_{1}$ to laminin etc. The specificity of integrins, however, is still a matter of speculation since several integrins can bind the same glycoproteins and since several glycoproteins can bind to the same integrin (Ruoslahti, 1991; Heino, 1993).

In the particular case of trophoblast, several studies (Damsky et al., 1992; Korhonen et al., 1991; Aplin, 1993; Burrows et al., 1993) including our own (Bischof et al., 1993) have shown that villous and intermediate cytotrophoblasts express different integrins. While villous cytotrophoblasts express predominantly the $\alpha_{6} \beta_{4}$ integrin (a probable laminin receptor, Lee et al. 1992) in a polarized manner (towards the basement membrane), intermediate cytotrophoblasts modulate their integrins: in the proximal region (cytotrophoblasts columns) they express $\alpha_{6} \beta_{4}$ in a non-polarized way whereas in the most distal part (the placental bed) they express the $\alpha_{5} \beta_{1}$ integrin, a fibronectin receptor. Thus, while cytotrophoblasts migrate from the villous into the decidua they modulate their integrin repertoire from being $\alpha_{6} \beta_{4}$ positive and $\alpha_{5} \beta_{1}$ negative to becoming $\alpha_{6} \beta_{4}$ negative and $\alpha_{5} \beta_{1}$ positive. This change in integrin expression goes along with the acquisition of an invasive phenotype.

Invasion is not due to passive growth pressure but to an active biochemical process. A cell is recognized to be invasive by virtue of its ability to secrete proteases and cytotrophoblasts are no exception (Bischof and Martelli, 1992). Cytotrophoblasts have been found to secrete metalloproteinases (Fisher $e t$ al., 1985, 1989; Bischof et al., 1991). These enzymes which are secreted as inactive proenzymes are grouped into three subfamilies according to their substrate specificities: collagenases, gelatinases and stromelysins (for review, see Matrisian, 1990). The collagenases digest collagen types I, II, III, VII and $X$ and are thus suited for digesting the collagens of the interstitium. The gelatinases are represented by two enzymes: the $72 \mathrm{kDa}$ gelatinase (gelatinase $\mathrm{A}$ ) and the $92 \mathrm{kDa}$ gelatinase (gelatinase $\mathrm{B}$ ). These matrix metalloproteinases digest collagen type IV (the major constituent of basement membranes) and denatured collagen (gelatin). The stromelysins have a much broader digestion spectrum and degrade fibronectin, laminin, collagens IV, V, VII and proteoglycans. Cytotrophoblasts essentially secrete gelatinases, and their invasive potential seems to be mediated by the $92 \mathrm{kDa}$ gelatinase (Librach et al., 1991).

Since it has been shown that the collagenolytic activity of cytotrophoblasts is specifically regulated by the glycoproteins of the extracellular matrix (Bischof et al., 1991; Emonard et al., 1990) and since this regulation could possibly occur through integrins (Werb et al., 1989, at least in rabbit skin fibroblasts), we decided to compare the gelatinolytic activity of villous and intermediate cytotrophoblasts and to see to what extent this proteolytic capacity was dependent on the type of integrins expressed on cytotrophoblasts.

\section{Materials and methods}

\section{Cell preparation and separation}

Cytotrophoblasts were prepared and purified according to a method already reported by us (Bischof et al., 1991). Briefly, trophoblastic tissue obtained from induced abortions (6-12 weeks of pregnancy) was digested with trypsin, separated from blood cells and syncytia on a discontinuous Percoll gradient and immunopurified by an antibody (anti-CD45) coupled to magnetic particles (Dyna beads; Dynal, Milian, Geneva, Switzerland) in order to eliminate all contaminating lymphomyeloid cells. These cytotrophoblasts were counted in a Neubauer haemocytometer in the presence of Trypan Blue (Sigma) and either put into culture as such $\left(10^{6}\right.$ cells $\left./ \mathrm{ml}\right)$ or separated further. In order to separate the purified cytotrophoblasts into HLA-G positive and negative cells, the purified cytotrophoblasts were resuspended $\left(5 \times 10^{6}\right.$ cells in $\left.2 \mathrm{ml}\right)$ in Dulbecco's modified Eagle's medium (DMEM; Gibco, Basel, Switzerland) containing $10 \%$ fetal bovine serum. The suspension was then incubated for $30 \mathrm{~min}$ at $4^{\circ} \mathrm{C}$ with $30 \mu \mathrm{l}$ of a mouse monoclonal antibody which detects a common framework epitope of histocompatibility-linked antigen (HLA)-A, B C (Dako-HLA-ABC, W6/32; Dako, IG Geneva, Switzerland). Since extravillous cytotrophoblasts do not express HLA-A, B or C (Hunt et al., 1990) but express HLA-G which is also recognized by the antibody W6/32, the cytotrophoblasts, positive for W6/32 will be called HLA-G positive hereafter.

After incubation the cells were washed with phosphatebuffered saline solution containing $0.1 \%$ bovine serum albumin (BSA, radioimmunoassay grade; Sigma). Prewashed (with phosphate-buffered saline solution and BSA) magnetic particles coated with a second antibody ( $30 \mu$ l Dyna beads) were then incubated with the cell suspension for $20 \mathrm{~min}$ at $4^{\circ} \mathrm{C}$. A magnet was then applied along the test tube to retain the particles bound to the cells expressing HLA-G on their surface. The supernatant (HLA-G negative cells) was poured into a clean test tube and cells counted (as mentioned above). The cells immobilized by the magnet (HLA-G positive) were washed and resuspended in culture medium.

The same separation technique was also used with primary antibodies to the $\alpha_{5}$ integrin subunit (CDw49e, clone SAM 1) and with antibodies to the $\alpha_{6}$ integrin subunit (CDw49f, clone $\mathrm{GoH} 3$; both antibodies from Immunotech, Marseille, France). This allowed us to obtain $\alpha_{6}$ positive and $\alpha_{6}$ negative as well as $\alpha_{5}$ positive and $\alpha_{5}$ negative cytotrophoblast suspensions. 


\section{Culture conditions}

Purified cytotrophoblasts $(200 \mu \mathrm{l})$, HLA-G positive and HLAG negative cytotrophoblasts, $\alpha_{5}$ positive and $\alpha_{5}$ negative cytotrophoblasts and $\alpha_{6}$ positive and $\alpha_{6}$ negative cytotrophoblasts $\left(10^{6} \mathrm{cells} / \mathrm{ml}\right)$ were incubated in duplicates in 12-well tissue culture plates (Costar, Cambridge, MA, USA) under a $5 \% \mathrm{CO}_{2}$ and $95 \%$ air atmosphere in an incubator at $37^{\circ} \mathrm{C}$. Certain wells were precoated with $300 \mu \mathrm{l}$ of $0.5 \%$ agarose in phosphate-buffered saline (Agar Noble Difco). The culture medium was DMEM containing $2 \mathrm{mmol} / \mathrm{l} \mathrm{L-glutamine} \mathrm{(Gibco),}$ $4.2 \mathrm{mmol} / \mathrm{l}$ magnesium sulphate, $25 \mathrm{mmol} / \mathrm{l}$ HEPES, $1 \%$ gentamycin, $1 \%$ amphotericin B, $100 \mu \mathrm{g} / \mathrm{ml}$ streptomycin and $100 \mathrm{IU} / \mathrm{ml}$ penicillin in the absence of serum. Medium was harvested on day 2 , and the culture stopped on day 5 . The supernatants were divided into aliquots and stored at $-20^{\circ} \mathrm{C}$ until assayed. The cells were lysed with $200 \mu \mathrm{l}$ of Triton X$100(2.5 \%$ in water $)$ and stored at $-20^{\circ} \mathrm{C}$.

\section{Hormone and protein assays}

Total human chorionic gonadotrophin (HCG $+\beta H C G$ ) was measured in the supernatants by a microparticle enzyme immunoassay with a sensitivity of $1 \mathrm{mIU} / \mathrm{ml}$ and a coefficient of variation of $3.6 \%$ (Abbott, Abbott Park, IL, USA). Fetal fibronectin was measured by a commercially available enzyme immunoassay with a sensitivity of $50 \mathrm{ng} / \mathrm{ml}$ and a coefficient of variation of $7.5 \%$ (Adeza Biochemical, Sunnyvale, CA, USA). Total cell proteins were measured by the Bio-Rad protein assay according to the manufacturer's instructions and using BSA as the standard (Bio-Rad, Munich, Germany).

\section{Gelatinase assays}

Qualitative assessment of the secreted gelatinases was performed by zymography as previously reported by us (Bischof et al:, 1991). Quantitative estimation of gelatinolytic activity in the culture supernatants was performed by measuring the degradation of heat-denatured radiolabelled collagen type IV, using a method modified from Emonard et al. (1990). Briefly, tritiated human type IV collagen $\left(N-\left[2,3-{ }^{3} \mathrm{H}\right]\right.$ propionate), sp. act. $0.0067 \mathrm{GBq} / \mathrm{mg}$; du Pont de Nemours, Geneva, Switzerland) was neutralized at $\mathrm{pH} 7.4$ with Tris- $\mathrm{HCl}$ buffer $(50 \mathrm{mM})$ containing $0.15 \mathrm{M} \mathrm{NaCl}, 4 \mathrm{mM} \mathrm{CaCl}{ }_{2}$ and incubated in a water bath at $60^{\circ} \mathrm{C}$ for 30 min to form gelatin. After cooling, $\mathrm{N}$-ethylmaleimide (NEM, $0.5 \mathrm{mM}$; Sigma) and phenylmethylsulphonyl fluoride (PMSF, 0.1 M; Sigma) were added to inhibit thiol and serine proteases respectively. This solution contained $10000 \mathrm{cpm}$ of radiolabelled gelatin per $100 \mu \mathrm{l}$. Collagenase (EC 3.4.24.3) from Clostridium histolyticum (330 U/mg; Sigma) was used as a standard. The standard curve ranged from 0.8 to $50 \mathrm{ng} / \mathrm{ml}(0.26-16.5 \mathrm{mU} / \mathrm{ml})$.

Aliquots of $100 \mu$ l of sample or standards were pipetted in duplicates into assay tubes in the presence or absence of $15 \mu \mathrm{l}$ of $0.4 \mathrm{M}$ ethylenediaminotetraacetate (EDTA, final concentration $25 \mathrm{mM}$ ). In order to activate the gelatinases to be measured, all tubes received $15 \mu \mathrm{l}$ of $2.4 \mathrm{mM} \mathrm{4-}$ aminophenylmercuric acetate (APMA; Sigma) in Tris buffer ( $0.05 \mathrm{M}$ Tris, $0.05 \%$ Triton X-100, $5 \mathrm{mM} \mathrm{CaCl}_{2}, 0.02 \% \mathrm{NaN}_{2}$, $\mathrm{pH} 7.0$ ) and the tubes were incubated at $25^{\circ} \mathrm{C}$ for $15 \mathrm{~min}$. Substrate $(100 \mu \mathrm{l}, 10000 \mathrm{cpm})$ was added and the tubes incubated at $37^{\circ} \mathrm{C}$ overnight. After incubation, the tubes were put on ice and undigested gelatin was precipitated by addition of $100 \mu \mathrm{l}$ of $36 \%$ trichloroacetic acid (TCA; Sigma) and incubated for $1 \mathrm{~h}$ at $4^{\circ} \mathrm{C}$. The incubation mixture was finally centrifuged at $6000 \mathrm{~g}$ for $25 \mathrm{~min}$ at $4^{\circ} \mathrm{C}$ and an aliquot of the supernatants $(100 \mu \mathrm{l})$ was added to $3 \mathrm{ml}$ of scintillation cocktail (Luma Gel; Medipro, Teufen, Switzerland) and the samples counted in a liquid scintillation counter (Packard) at an efficiency of $35 \%$. The gelatinolytic activity of the cell supernatants was calculated by comparison to the standard curve run in parallel and expressed (as mean of duplicates in the absence of EDTA minus mean of duplicates in the presence of EDTA) in ng per mg total cell proteins or ng per $10^{6}$ cells.

\section{Adhesion assay}

Cytotrophoblasts and the different cytotrophoblast subsets were cultured for 2 days (as described above) in 12-well tissue culture plates (Costar, Cambridge, MA, USA) precoated $(50 \mu \mathrm{g} /$ $\mathrm{ml}$ for $4 \mathrm{~h}$ at room temperature) with fibronectin (Boehringer, Mannheim, Germany), laminin (Sigma), BSA (Sigma), agarose (as described above) and Matrigel ( $200 \mu \mathrm{l}$, diluted 1/5; Collaborative Research, Inotech, Switzerland). After culture, the supernatants were aspirated and the unattached cells pelleted by centrifugation. The attached and unattached cells were lysed by water and by two freezing/thawing cycles. Total DNA was measured by fluorimetry and compared to a plasmid DNA of known concentration. Results were expressed as percentage of DNA of attached cells versus DNA of total cells (attached and non-attached).

\section{Immunohistochemistry}

Aliquots of. cytotrophoblasts, HLA-G negative, $\alpha_{5}$ negative and $\alpha_{6}$ negative cytotrophoblasts were taken before the cells were put into culture. The aliquots were centrifuged at $700 \mathrm{~g}$ onto glass slides with a cytospin centrifuge (Shandon, IG, Geneva, Switzerland). They were fixed in acetone for $5 \mathrm{~min}$ at $-20^{\circ} \mathrm{C}$ and stained as described previously (Bischof et al., 1991) using anti-CD45, anti HLA-G, anti-CDw49e and antiCDw49f as primary antibodies (dilution 1:20). The slides were evaluated under the microscope and the results expressed as percentage of positive cells per 300 total cells counted.

\section{Results}

\section{Immunohistochemistry}

As shown in Table I, purified cytotrophoblasts (after immunoadsorption of $\mathrm{CD} 45$ positive cells) were still contaminated by

Table I. Immunohistochemical study on cell separation (percentage of positive cells, mean $\pm \mathrm{SEM}, n=3$ )

\begin{tabular}{llll}
\hline Antibodies & \multicolumn{2}{l}{ Cytotrophoblasts } & \\
\cline { 2 - 4 } & Purified & $\alpha_{6}$ negative & $\alpha_{5}$ negative \\
\hline Anti-CD45 & $3.0 \pm 0.5$ & - & - \\
Anti-HLA-G & $29.6 \pm 8.6$ & - & - \\
Anti- $\alpha_{6}$ & $30.5 \pm 8.1$ & $6.4 \pm 0.8$ & $27.9 \pm 7.1$ \\
Anti- $\alpha_{5}$ & $19.2 \pm 1.8$ & $30.5 \pm 8.1$ & $4.4 \pm 0.3$ \\
\hline
\end{tabular}


$-3 \%$ of lymphomyeloid cells. About one-third of the cells expressed HLA-G and one-third expressed the integrin subunit $\alpha_{6}$, whereas $19.2 \%$ of the purified cytotrophoblasts expressed the $\alpha_{5}$ subunit. After separation of the cells with anti-integrin antibodies, the $\alpha_{6}$ negative cytotrophoblasts still contained $\sim 6 \%$ of cells expressing the $\alpha_{6}$ integrin subunit, whereas only $30.5 \%$ of them expressed the $\alpha_{5}$ subunit. Similarly, the $\alpha_{5}$ negative cytotrophoblasts were still contaminated by $4.4 \%$ of cells expressing the $\alpha_{5}$ subunit, and $27.9 \%$ of the $\alpha_{5}$ negative cells expressed the $\alpha_{6}$ subunit.

Thus, the separation technique with the anti- $\alpha_{5}$ or anti- $\alpha_{6}$ antibodies and the magnetic particles allowed a relatively good separation of cells expressing the integrin $\alpha_{5}$ or $\alpha_{6}$, but left $\sim 4-6 \%$ of positive cells in the fractions which were supposed to be negative for these integrins. Immunohistochemistry for the $\alpha_{5}$ and $\alpha_{6}$ integrin subunits could not be performed on the positive cells since these cells were already saturated with the antibodies and the magnetic particles used during the separation procedure.

\section{Proteolytic activity of cytotrophoblasts}

In the absence of APMA, which activates the progelatinases into active gelatinases even when bound to tissue inhibitor of metalloproteinases (TIMP), the assay measures the gelatinases secreted as active enzymes. These were below the sensitivity of the assay $(0.4 \mathrm{ng} / \mathrm{ml})$ for cytotrophoblasts grown for 2 days on agarose or plastic (Figure 1). Cytotrophoblasts grown on Matrigel released low but measurable amounts of active gelatinases $(0.5+0.02 \mathrm{ng} / \mathrm{ml}, \mathrm{SD})$.

In the absence of EDTA but in the presence of APMA, the test measures the total gelatinolytic activity due to metalloproteinases but also to other enzymes. The secreted activity was high for all cytotrophoblasts irrespective of the substrate on which the cells were grown.

The gelatinase activity measured in the presence of both APMA and EDTA (APMA is inhibited by EDTA) represents the secreted activity of in-vitro activated prometalloproteinases (called gelatinolytic activity hereafter). This activity was significantly higher in supernatants of cytotrophoblasts grown on Matrigel or plastic ( $P<0.01, P<0.05$ respectively) as compared to cells grown on agarose (Figure 1).

As shown in Figure 2, after immunopurification of the cytotrophoblasts with antibodies to CD45 (to remove lymphomyeloid cells), their secreted gelatinolytic activity was significantly higher $(P<0.05)$ than before immunopurification. This difference could be observed irrespective of the substrate on which the cells were grown.

\section{Adhesion assay}

Irrespective of the cytotrophoblasts subset, attachment of the cells to agarose or to BSA was very weak (6-20\%, Figure 3). Attachment of cytotrophoblasts to fibronectin or to laminin (48.3 and $45.9 \%$ respectively) was significantly weaker ( $P$ $<0.05$ ) than to plastic $(88.0 \%)$. As compared to purified cytotrophoblasts, the number of $\alpha_{5}$ positive cytotrophoblasts and $\alpha_{5}$ negative cytotrophoblasts attaching to plastic or Matrigel was significantly reduced $(P<0.05$ to $P<0.001$, Figure 3 ).

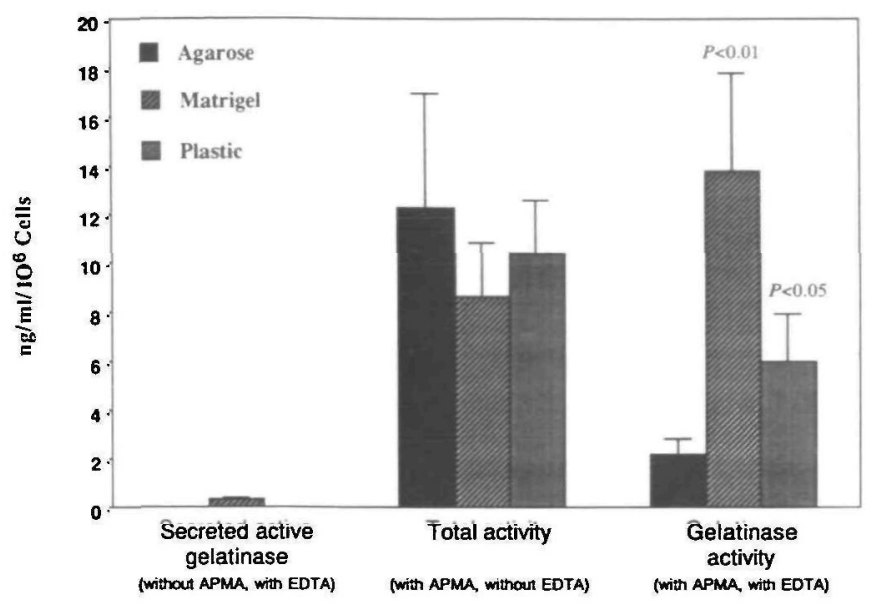

Figure 1. Gelatinolytic activities secreted by purified cytotrophoblasts grown for 2 days on agarose, Matrigel or plastic $(n=4$; mean $\pm \mathrm{SD})$. APMA $=4$-aminophenylmercuric acetate; EDTA $=$ ethylenediaminotetraacetate.

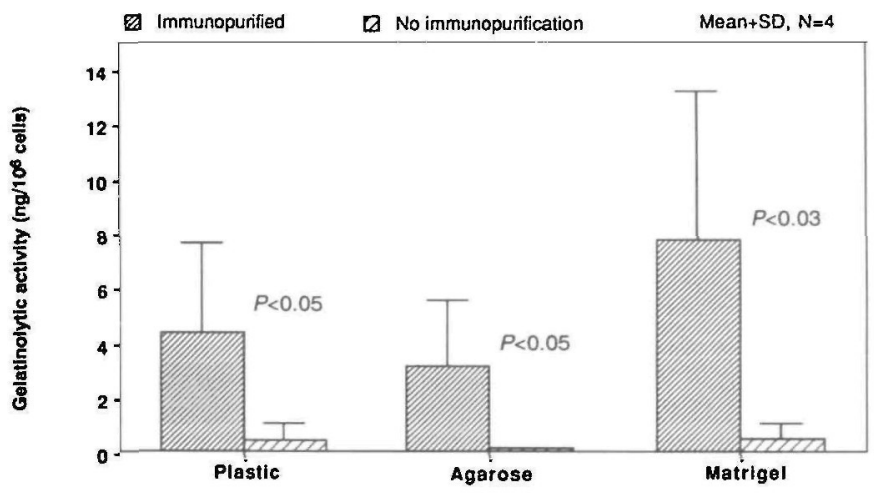

Figure 2. Gelatinolytic activity secreted by cytotrophoblasts grown for 2 days on agarose, Matrigel or plastic. Comparison between purified (with CD45) and non-purified cells.

Attachment of $\alpha_{5}$ negative cells to fibronectin and to laminin (29.6 and $23.7 \%$ respectively) was significantly reduced when compared to $\alpha_{5}$ positive cytotrophoblasts (67.5 and $48.2 \%, P$ $<0.05$ and $P<0.001$ respectively). The number of $\alpha_{6}$ positive cytotrophoblasts which attached to plastic $(50.4 \%)$ was significantly lower $(P<0.03)$ than purified cytotrophoblasts (88.0\%). Attachment of $\alpha_{6}$ negative cytotrophoblasts to laminin was significantly $(P<0.05)$ reduced as compared to purified cytotrophoblasts (16.9 versus $45.9 \%$, Figure 3 ).

\section{Separation of cytotrophoblasts by HLA-G}

When grown on plastic, HLA-G positive cells secreted significantly $(P<0.05)$ higher amounts of gelatinolytic activity than HLA-G negative cells (209 and $19.5 \mathrm{ng} / \mathrm{mg}$ protein respectively, Figure 4). HLA-G negative cells grown on agarose secreted significantly more $(P<0.05)$ gelatinases than the same cells grown on plastic $(31.8$ and $19.5 \mathrm{ng} / \mathrm{mg}$ protein respectively). HLA-G positive or negative cells, irrespective of the substrate on which they grew, secreted similar concentrations of HCG. 

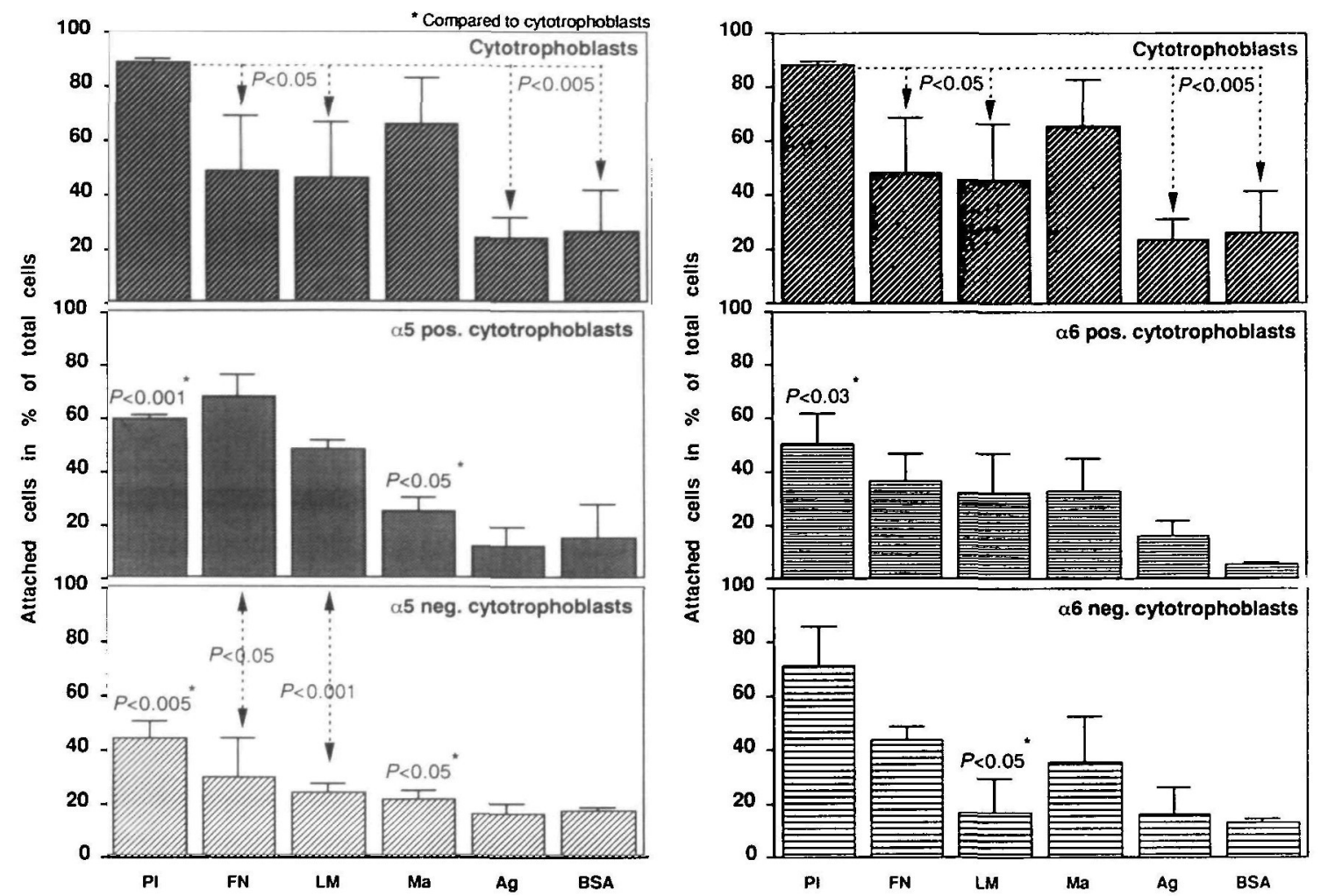

Figure 3. Attachment of different cytotrophoblast subsets after $48 \mathrm{~h}$ of culture on plastic (PI), fibronectin (FN), laminin (LM), Matrigel $(\mathrm{Ma})$, agarose $(\mathrm{Ag})$ and bovine serum albumin $(\mathrm{BSA})$. Values are mean $\pm \mathrm{SD}$ of three different cultures. Pos. $=$ positive; neg. $=$ negative.

\section{Separation of cytotrophoblasts by integrins}

As can be seen on the zymogram (Figure 5), $\alpha_{6}$ positive cytotrophoblasts expressed a much higher proteolytic activity than $\alpha_{6}$ negative cytotrophoblasts, irrespective of the substrate on which they grew. The major digestion band had a molecular size of $92 \mathrm{kDa}$. A minor faint digestion band with a molecular size $>200 \mathrm{kDa}$ was also visible. Similar results were observed when supernatants of $\alpha_{5}$ positive and negative cells were compared (results not shown).

The gelatinolytic activity secreted by $\alpha_{6}$ positive cytotrophoblasts grown on agarose or plastic was significantly higher $(P$ $<0.03$ to $P<0.001)$ than the activity secreted by purified cytotrophoblasts or by the other cytotrophoblast subsets $\left(\alpha_{6}\right.$ negative, $\alpha_{5}$ positive, $\alpha_{5}$ negative). Compared to agarose, $\alpha_{6}$ positive cytotrophoblasts grown on plastic secreted a significantly $(P<0.05)$ higher gelatinolytic activity. In addition, $\alpha_{5}$ positive cells secrete a significantly $(P<0.05$ on plastic, $P<0.03$ on agarose) higher gelatinolytic activity than $\alpha_{5}$ negative cells (Figure 6).

Despite the fact that adhesion of the cells to plastic increased significantly $(P<0.05)$ their fetal fibronectin secretion, $\alpha_{6}$ positive cytotrophoblasts secreted significantly less fetal fibronectin than $\alpha_{5}$ positive cytotrophoblasts $(P<0.03$ on agarose, $P<0.05$ on plastic). Furthermore, the fetal fibronectin secretion of $\alpha_{5}$ positive cytotrophoblasts was significantly higher that of $\alpha_{5}$ negative cytotrophoblasts or of purified cytotrophoblasts ( $P<0.03, P<0.05$ respectively, Figure 6 ).

In contrast, $\alpha_{6}$ positive and $\alpha_{5}$ positive cells secreted similar
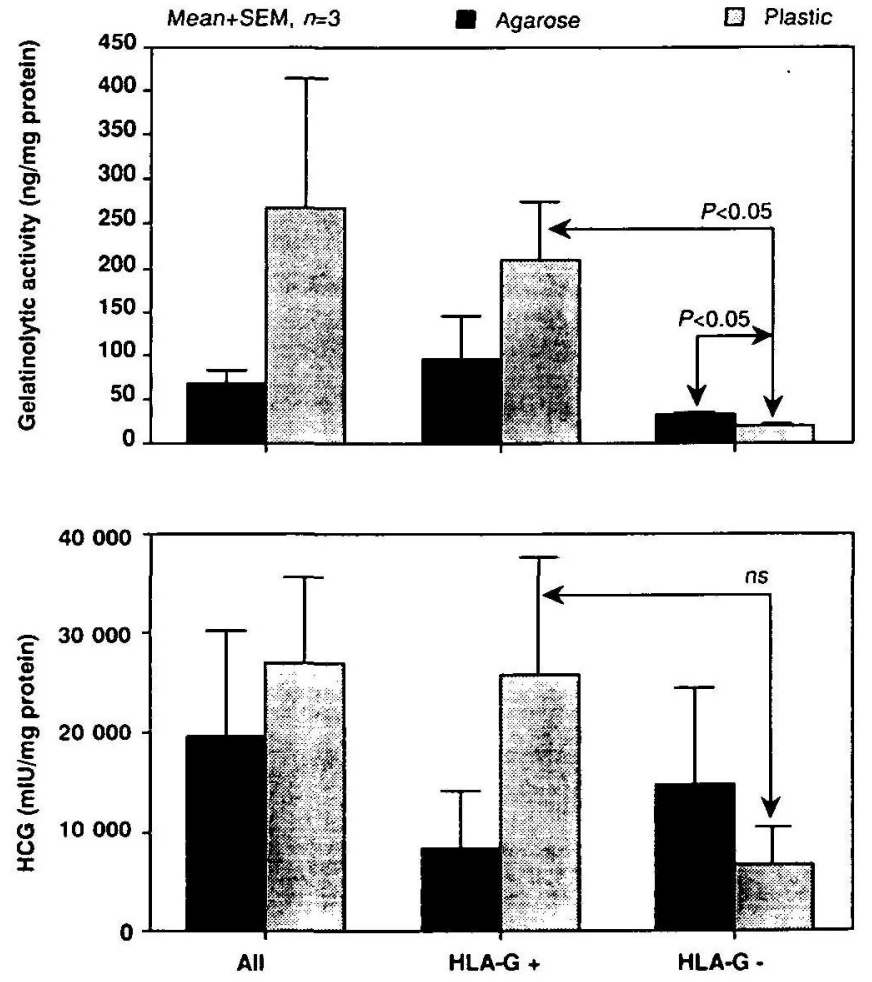

Figure 4. Gelatinolytic activity and human chorionic gonadotrophin (HCG) secretion of purified cytotrophoblasts (all), HLA-G positive cytotrophoblasts (HLA-G + ) and HLA-G negative cytotrophoblasts (HLA-G-) grown in culture for $48 \mathrm{~h}$. 
Ag Plastic Matrigel Agarose

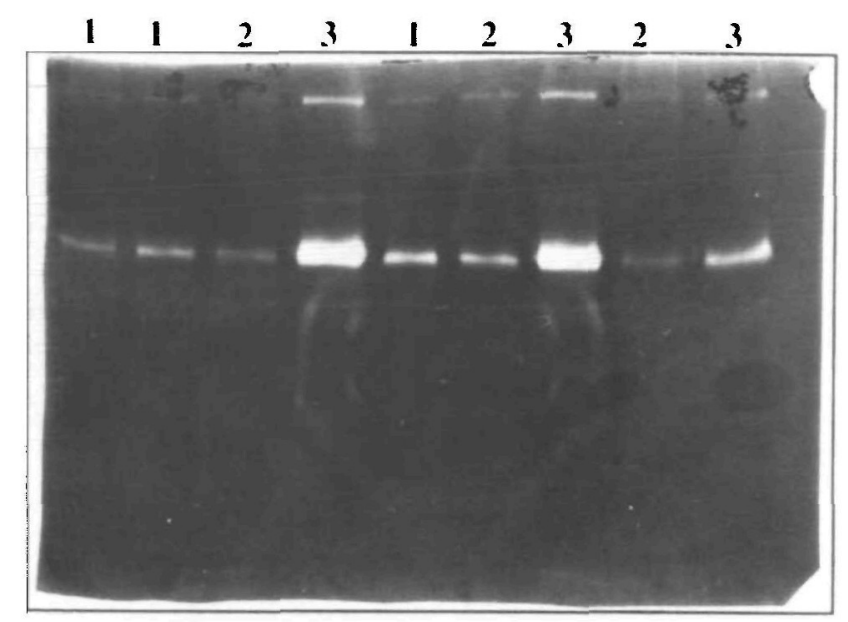

1. Cytotrophoblast cells $(1106 / \mathrm{ml})$

2. $\alpha 6$ integrin-free cytotrophoblast cells

3. $\alpha 6$ integrin-positive cytotrophoblast cells

Figure 5. Zymogram of 2 day culture supernatants from cytotrophoblast subsets grown on agarose $(\mathrm{Ag})$, plastic or Matrigel.

amounts of HCG. Adhesion of these cells to plastic, however, increased significantly $(P<0.05)$ their HCG release as compared to cells grown on agarose. Finally, $\alpha_{5}$ positive cytotrophoblasts grown on plastic secreted significantly $(P<$ 0.05 , Figure 6) more HCG than $\alpha_{5}$ negative cytotrophoblasts or purified cytotrophoblasts grown on plastic. In summary, $\alpha_{6}$ positive cytotrophoblasts secreted large amounts of gelatinolytic activity and low concentrations of fetal fibronectin, whereas $\alpha_{5}$ positive cytotrophoblasts secreted small amounts of gelatinolytic activity and high concentrations of fetal fibronectin; their HCG secretion was not significantly different.

\section{Comparison of gelatinolytic activity secretion rates}

Figure 7 compares, for all cytotrophoblast subsets, their localization in the anchoring villous (from Korhonen et al., 1991; Damsky et al., 1992; Aplin 1993; Burrows et al., 1993; Bischof et al., 1993) together with their gelatinolytic activity secretion rate expressed as the activity secreted per $\mathrm{mg}$ protein and per $24 \mathrm{~h}$ over a period of 5 days in culture. Cytotrophoblasts, HLA-G positive and $\alpha_{5}$ positive cytotrophoblasts secreted gelatinases at a similar rate to purified cytotrophoblasts. In contrast, $\alpha_{6}$ positive cytotrophoblasts secreted gelatinases at a significantly higher rate $(P<0.03)$ compared with $\alpha_{5}$ positive cytotrophoblasts. As compared to their positive counterparts or to purified cytotrophoblasts (data not shown), HLA-G negative, $\alpha_{5}$ negative and $\alpha_{6}$ negative cytotrophoblasts secreted gelatinases at a significantly lower rate $(P<0.05$, Figure 7).

\section{Discussion}

The population of cytotrophoblasts used here was purified by immunoadsorption with an antibody to CD45 and with a magnetic particle separation. This technique yielded a $97 \%$

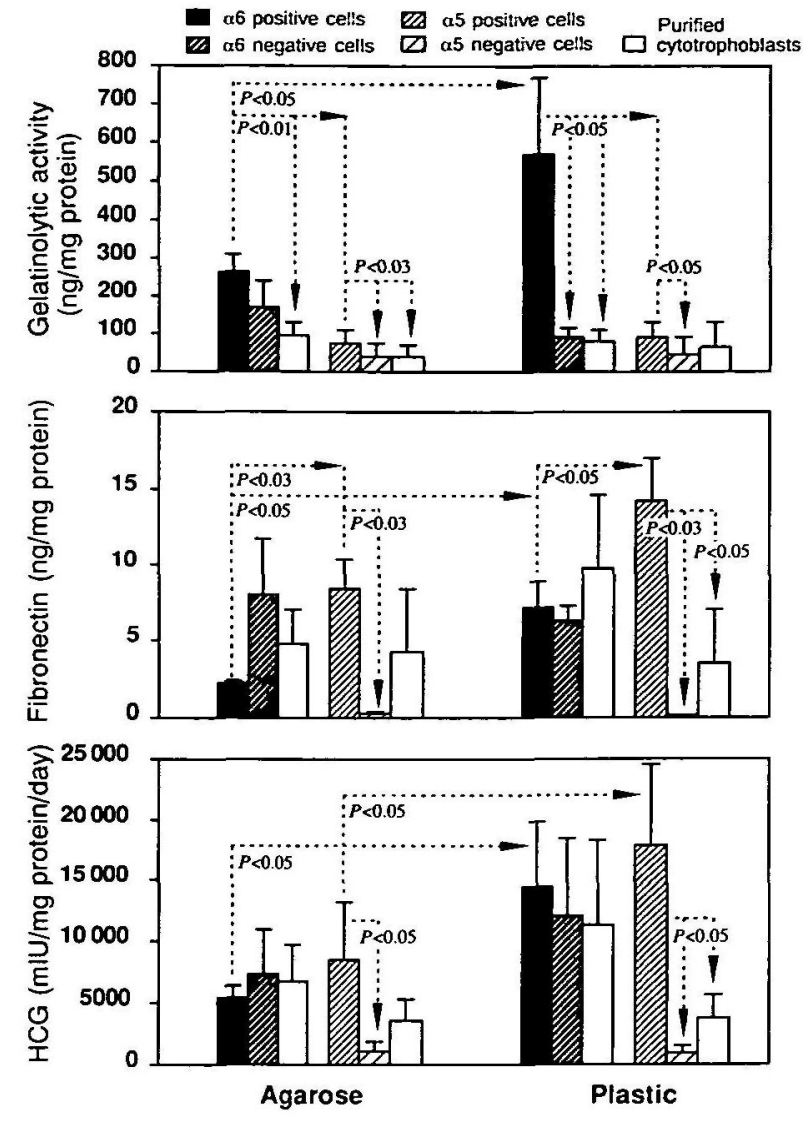

Figure 6. Gelatinolytic activity, fibronectin and human chorionic gonadotrophin (HCG) secretion of different cytotrophoblast subsets grown for $48 \mathrm{~h}$ on agarose or plastic. Values are mean $\pm \mathrm{SD}$ of three different cultures.

pure cytotrophoblast preparation with a $3 \%$ contamination by lymphomyeloid cells. Using the same technique to separate cytotrophoblasts according to the integrins they express, the same sort of results were observed: $4-6 \%$ of cells were left behind.

As reported by us here and previously (Bischof et al., 1991), purified cytotrophoblasts grown on agarose do not attach to this substrate at all or do so very poorly, but remain viable and floating in the supernatant. Purified cytotrophoblasts grown on Matrigel and plastic do attach. These cytotrophoblasts did not secrete active gelatinases when grown on agarose or plastic, but released small amounts of active gelatinases when grown on Matrigel. Thus gelatinases are secreted by cytotrophoblasts mainly in their inactive proenzyme form. Binding of the cells to Matrigel or plastic activated their secretion of gelatinases, whereas when the cells remained in suspension (as on agarose), their secretion of gelatinases was low. This confirms previous reports where these same differences were also observed, either semi-quantitatively on zymograms (Bischof et al., 1991) or quantitatively with a similar assay to ours (Emonard et al., 1990). Discarding contaminating lymphomyeloid cells from the cytotrophoblast suspension had a marked effect on their gelatinolytic activity, irrespective of the substrate on which the cells grew. Since immunopurified cytotrophoblasts secreted significantly more gelatinolytic activity than non-purified cells, 


\section{P.Bischof et al.}

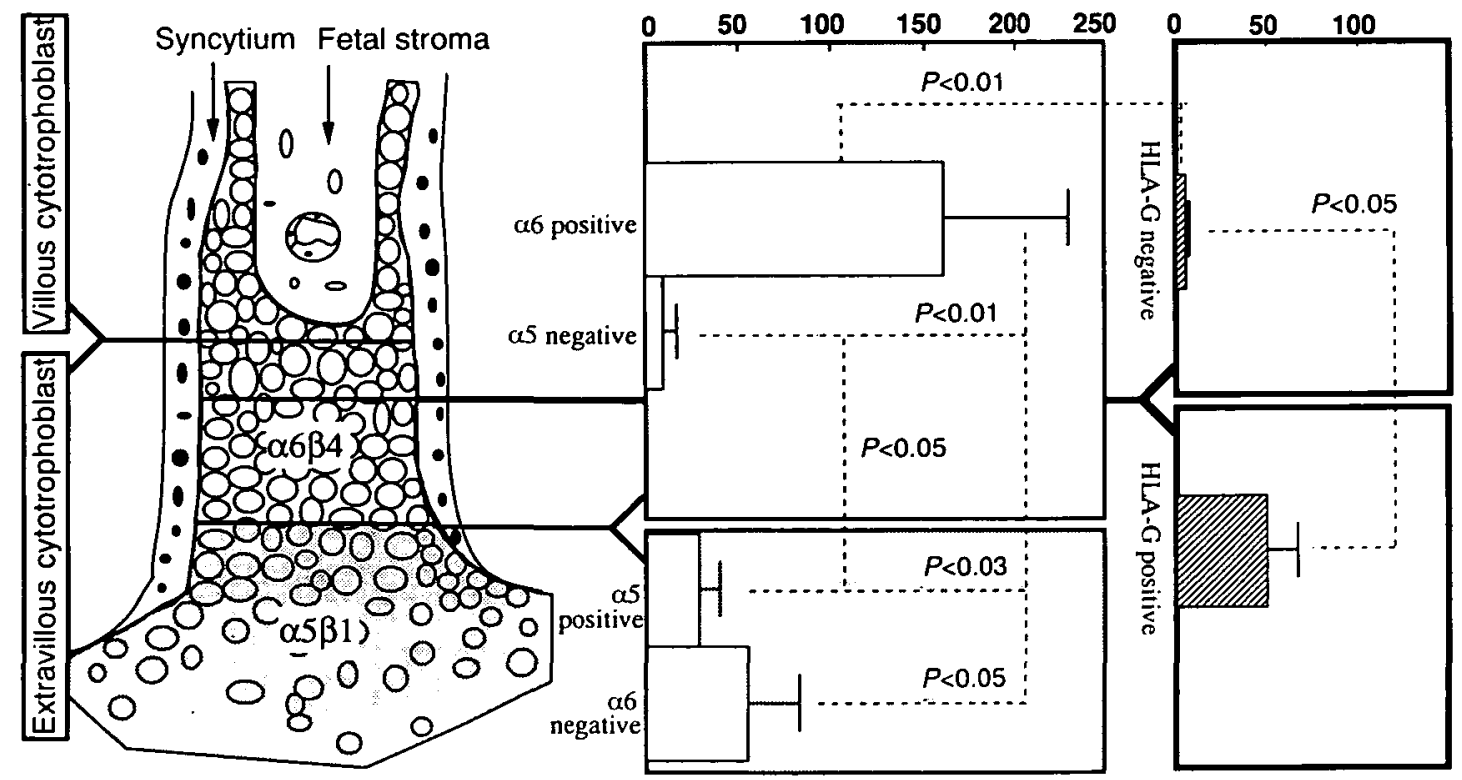

Figure 7. Comparison between localization of different cytotrophoblast subsets and their secretion rates of gelatinolytic activity as measured over 5 days in culture (mean $\pm \mathrm{SD}, n=3$ ).

one must assume that contaminating lymphomyeloid cells did secrete factors which inhibited cytotrophoblast gelatinases. TIMP is a potent inhibitor of gelatinases which is produced by lymphomyeloid cells such as monocytes (Opdenakker et al., 1991) and macrophages (Shapiro et al., 1993). It could thus be that the lower gelatinolytic activity secreted by cytotrophoblasts in the presence of lymphomyeloid cells was possibly due to monocyte-macrophage-derived TIMP.

One-third of the purified cytotrophoblasts were HLA-G positive, one-third were positive for the $\alpha_{6}$ integrin subunit and about one-fifth were positive for the $\alpha_{5}$ subunit. An unknown proportion of the $\alpha_{6}$ positive cytotrophoblasts must be villous cytotrophoblasts since these express also the $\alpha_{6}$ integrin subunit (Korhonen et al., 1991; Damsky et al., 1992; Aplin, 1993; Burrows et al., 1993; Bischof et al., 1993). Extravillous cytotrophoblasts express both $\alpha_{5}$ or $\alpha_{6}$ integrin subunits, depending on their location within the placental bed (Korhonen et al., 1991; Damsky et al., 1992; Aplin, 1993; Burrows et al., 1993; Bischof et al., 1993). Thus the $\alpha_{6}$ positive cytotrophoblasts used here represented a mixture of villous and extravillous cytotrophoblasts, whereas the $\alpha_{5}$ positive cytotrophoblasts were purely extravillous cells. The extravillous trophoblast of first-trimester anchoring villi expresses both HLA-G protein (Shorter et al., 1993) and mRNA (Chumbley et al., 1993) together with the $\alpha_{5}$ or $\alpha_{6}$ integrin subunits. The cytotrophoblasts located in the proximal portion of the cell columns do not express HLA-G (Shorter et al., 1993) but are $\alpha_{6}$ positive (Korhonen et al., 1991; Damsky et al., 1992; Aplin, 1993; Burrows et al., 1993; Bischof et al., 1993). Thus, $\alpha_{6}$ positive cytotrophoblasts and HLA-G positive cytotrophoblasts are not the same cell population but are overlapping populations. In our hands, the purely extravillous cytotrophoblast population (HLA-G positive) secreted a significantly higher gelatinolytic activity than villous cytotrophoblasts (HLA-G negative). Since the invasive behaviour of a cell depends on its secretion of gelatinases (Mignatti and Rifkin, 1993), our observation could explain why extravillous cytotrophoblasts are invasive whereas villous cytotrophoblasts are not. It is interesting to note in this context that cytotrophoblasts positive for the $\alpha_{5}$ integrin subunit are also exclusively extravillous cytotrophoblasts (Korhonen et al., 1991; Damsky et al., 1992; Aplin, 1993; Burrows et al., 1993; Bischof et al., 1993). These cells secrete a gelatinolytic activity which was not different from HLA-G positive cytotrophoblasts but which was significantly higher than HLA-G negative cytotrophoblasts.

The $\alpha_{6}$ positive cytotrophoblasts expressed a gelatinolytic activity which was significantly higher than $\alpha_{5}$ positive, $\alpha_{5}$ negative or $\alpha_{6}$ negative cytotrophoblasts. This cell population is a mixture of villous and extravillous cytotrophoblasts. Since villous cytotrophoblasts (HLA-G negative) expressed a very low gelatinolytic activity one must admit that the high activity secreted by the $\alpha_{6}$ positive cells was mainly due to the extravillous $\alpha_{6}$ positive cytotrophoblasts. The $\alpha_{5}$ negative cytotrophoblast population is also a mixture of villous and extravillous cytotrophoblasts and should theoretically contain all $\alpha_{6}$ positive cytotrophoblasts. In fact, these represent only $30 \%$ of the $\alpha_{5}$ negative population. This might explain why the gelatinolytic activity of $\alpha_{5}$ negative cytotrophoblasts was significantly smaller than that of the $\alpha_{6}$ positive cytotrophoblasts. Obviously, cytotrophoblasts expressing neither $\alpha_{6}$ nor $\alpha_{5}$ were also present in the $\alpha_{5}$ negative cytotrophoblasts suspension. An alternative explanation for the difference in the gelatinolytic activity between $\alpha_{5}$ negative and $\alpha_{6}$ positive cytotrophoblasts could relate to the use of $\alpha_{6}$ antibodies used to isolate the cells. Function-perturbing anti-integrin antibodies have been shown to induce collagenases (Werb et al., 1989). We cannot rule out the possibility that our $\alpha_{6}$ antibody has induced the secretion of gelatinases. However, this is certainly not the case with the $\alpha_{5}$ antibody ( $\alpha_{5}$ positive cells had a low 
gelatinolytic activity). Furthermore, the anti- $\alpha_{6}$ and anti- $\alpha_{5}$ antibodies we used do not seem to be function-perturbing antibodies since the attachment of $\alpha_{6}$ positive cytotrophoblasts or $\alpha_{5}$ positive cytotrophoblasts to laminin or fibronectin was similar to that of purified cytotrophoblasts.

Compared to the $\alpha_{5}$ positive cytotrophoblasts, the extravillous $\alpha_{6}$ positive cytotrophoblasts are located more proximally to the villous stem, whereas the $\alpha_{5}$ positive cytotrophoblasts are localized more distally in the placental bed (Korhonen et al., 1991; Damsky et al., 1992; Aplin, 1993; Burrows et al., 1993; Bischof et al., 1993). Our results thus suggest that once the cytotrophoblasts have reached the placental bed and express the $\alpha_{5}$ integrin subunit, their gelatinolytic activity is lower than when they express the $\alpha_{6}$ integrin subunit and migrate along the cell columns. Since the $\alpha_{5}$ positive cytotrophoblasts secrete significantly higher concentrations of fibronectin than $\alpha_{6}$ positive cytotrophoblasts, one could postulate that once cytotrophoblasts express the $\alpha_{5} \beta_{1}$ integrin, their invasive behaviour and gelatinase secretion have almost stopped and the cells become immobile and secrete fibronectin. In contrast, extravillous $\alpha_{6}$ positive cytotrophoblasts are invasive and motile cells which secrete large amounts of gelatinases but little fibronectin.

As shown by immunohistochemistry (Korhonen et al., 1991; Damsky et al., 1992; Aplin, 1993; Burrows et al., 1993; Bischof et al., 1993) the $\alpha_{6} \beta_{4}$ integrin in villous cytotrophoblasts has a focal distribution along the villous basement membrane, whereas this integrin is not clustered on extravillous $\alpha_{6}$ positive cytotrophoblasts. Since clustering of integrins occurs upon binding to its ligand (Kornberg et al., 1992), focally distributed integrins are thus observed on immobile, anchored cells. Therefore it seems reasonable to suppose that villous cytotrophoblasts become motile and invasive when they depolarize their $\alpha_{6} \beta_{4}$ integrins. If this is correct, then one must admit that depolarization of the $\alpha_{6} \beta_{4}$ integrin is accompanied by an up-regulation of the gelatinolytic activity and a down-regulation of the fibronectin secretion. How these events might be connected is unknown. Since Werb et al. (1989) elegantly demonstrated in rabbit synovial fibroblasts that collagenase induction was a result of transduction through the $\alpha_{5} \beta_{1}$ integrin, one might speculate that gelatinase expression in extravillous cytotrophoblasts might be turned on and fibronectin secretion turned off by a lack of transduction through the $\alpha_{6} \beta_{4}$ integrin due to the depolarization of this integrin.

\section{Acknowledgements}

This work was supported by a grant (no. 32-39307.93) from the Swiss National Fund for Scientific Research to P.B.

\section{References}

Aplin, J.D. (1993) Expression of integrin $\alpha 6 \beta 4$ in human trophoblast and its loss from extra villous cells. Placenta, 14, 203-215.

Bischof, P. and Martelli, M. (1992) Proteolysis in the penetration phase of the implantation process. Placenta, 13, 17-24.

Bischof, P., Friedli, E., Martelli, M. and Campana, A. (1991) Expression of extracellular matrix-degrading metalloproteases by cultured human cytotrophoblast cells: effect of cell adhesion and immunopurification. Am. J. Obstet. Gynecol., 65, 1791-1801.
Bischof, P., Redard, M., Gindre, P., Vassilakos, P. and Campana, A. (1993) Localisation of $\alpha 2, \alpha 5$ and $\alpha 6$ integrin subunits in human endometrium, decidua and trophoblast. Eur. J. Obstet., Gynecol. Reprod. Biol., 51, 217-226.

Burrows, T.D., King, A. and Loke, Y.W. (1993) Expression of integrins by human trophoblast and differential adhesion to laminin and fibronectin. Hum. Reprod., 8, 475-484.

Chumbley, G., King, A., Holmes, N. and Loke, Y.W. (1993) In situ hybridization and nothern blot demonstration of HLA-G mRNA in human trophoblast populations by locus specific oligonucleotides. Hum. Immunol., 37, 7-22.

Damsky, C.H., Fitzgerald, M. and Fisher, S.J. (1992) Distribution patterns of extra cellular matrix components and adhesion receptors are intricately modulated during first trimester cytotrophoblast differentiation along the invasive pathway in vivo. J. Clin. Invest., 89, 210-222.

Emonard, H., Christiane, Y., Smet, M., Grimaud, J.A. and Foidart, J.M. (1990) Type IV and interstitial collagenolytic activities in normal and malignant trophoblast cells are specifically regulated by the extra cellular matrix. Invasion Metast., 10, 170-177.

Enders, A. (1968) Fine structure of anchoring villi of the human placenta. Am. J. Anat., 22, 419-452.

Fisher, S.J., Leitch, M.S., Kantor, M.S., Basbaum, C.B. and Kramer, R.H. (1985) Degradation of extracellular matrix by the trophoblastic cells of first trimester placentas. J. Cell. Biochem., 27, 31-41.

Fisher, S.J., Cui, T., Zhang, L., Hartmann, L., Grahl, K., Guo-Yang, Z., Tarpey, J. and Damsky, C.H. (1989) Adhesive and degradative properties of human placental cytotrophoblast cells in vitro. J. Cell Biol., 109, 891-902.

Genbacev, O., DeMesy Jensen, K., Schubach Powlin, S. and Miller, K. (1993) In vitro differentiation and ultrastructure of human extravillous trophoblast (EVT) cells. Placenta, 14, 463-475.

Heino, J. (1993) Integrin type extracellular matrix receptors in cancer and inflammation. Ann. Med., 25, 335-342.

Hunt, J.S., Fishback, J.L., Chumbley, G. and Loke, Y.W. (1990). Identification of class I MHC mRNA in human first trimester trophoblast cells by in situ hybridization. J. Immunol., 144, 44204425.

Kao, L.C., Caltabiano, S., Wu, S., Strauss, J.F. and Kliman, H. (1988) The human villous cytotrophoblast interaction with extra cellular matrix proteins, endocrine function and cytoplasmic differentiation in the absence of syncytium formation. Dev. Biol., 130, 693-702.

Khong, T.Y., De Wolf, F., Robertson, W.B. and Brossens, I. (1986) Inadequate maternal vascular response to placentation in pregnancies complicated by pre-eclampsia and by small-forgestational-age infant. Br. J. Obstet. Gynaecol., 93, 1049-1059.

Korhonen, M., Ylènne, J., Laitineen, L., Cooper, H.M., Quaranta, V. and Virtanen, I. (1991) Distribution of the alphal-beta6 integrin subunits in human developing and term placenta. Lab. Invest., 65 , 347-356.

Kornberg, L., Earp, H.S., Parson, J.T., Schaller, M. and Juliano, R.L. (1992) Cell adhesion or integrin culstering increases phosphorylation of a focal adhesion-associated tyrosine kinase. J. Biol. Chem., 267, 23439-23442.

Lee, E.C., Lotz, M.M., Steele, G.D. and Mercurio, A.M. (1992) The integrin $\alpha 6 \beta 4$ is a laminin receptor. J. Cell Biol., 117, 671-678.

Librach, C.L., Werb, Z., Fitzgerald, M.L., Chiu, K., Corwin, N.M., Esteves, R.A., Grobelny, D., Galardy, R. and Damsky, C.H. (1991) $92 \mathrm{kDa}$ type IV collagenase mediates invasion of human cytotrophoblasts. J. Cell Biol., 113, 437-449.

Matrisian, L. (1990) Metalloproteinases and their inhibitors in matrix remodelling. Trends Genet., 6, 121-125.

Mignatti, P. and Rifkin, D.B. (1993) Biology and biochemistry of proteases in tumour invasion. Physiol. Rev., 73, 161-195.

Mühlhauser, J., Crescimanno, C., Kaufmann, P., Höfler, H., 


\section{P.Bischof et al.}

Zaccheo, D. and Castellucci, M. (1993) Differentiation and proliferation pattern in human trophoblast revealed by c-erb B-2 oncogene product and EGF-R. J. Histochem. Cytochem., 41, 165-173.

Opdenakker, G., Masure, S., Proost, P., Billiau, A. and Van Damme, J. (1991) Natural human monocytes gelatinase and its inhibitor. FEBS Lett., 284, 73-78.

Ruoslahti, E. (1991) Integrins. J. Clin. Invest., 87, 1-5.

Shapiro, S.D., Kobayashi, D.K., Pentland, A.P. and Welgus, H.G. (1993) Induction of macrophage metalloproteinases by extra cellular matrix. J. Biol. Chem., 268, 8170-8175.

Shorter, S.C., Starkey, P.M., Ferry, B.L., Clover, L.M., Sargent, I.L. and Redman, C.W.G. (1993) Antigenic heterogeneity of human cytotrophoblast and evidence for the transient expression of $\mathrm{MHC}$ class I antigens distinct from HLA-G. Placenta, 14, 571-582.

Weitlauf, H.M. (1988) Biology of implantation. In Knobil, E. and Neil, J.D. (eds), Physiology of Reproduction. Raven Press, New York, pp. 231-262.

Werb, Z., Tremble, P.M., Behrendtsen, O., Crowley, E. and Damsky, C.H. (1989) Signal transduction through the fibronectin receptor induces collagenases and stromelysin gene expression. $J$. Cell Biol., 109, 877-889.

Zhou, Y., Damsky, C.H., Chiu, K., Roberts, J.M. and Fisher, S.J. (1993) Pre-eclampsia is associated with abnormal expression of adhesion molecules by invasive cytotrophoblasts. J. Clin. Invest., 91, 950-960.

Received on October 3, 1994; accepted on January 2, 1995 